\title{
Efficacy of flecainide, sotalol, and verapamil in the treatment of right ventricular tachycardia in patients without overt cardiac abnormality
}

\author{
Jaswinder S Gill, Davendra Mehta, David E Ward, A John Camm
}

\begin{abstract}
Objective-A comparison of the efficacy of verapamil, sotalol, and flecainide to suppress right ventricular tachycardia (VT) in patients with a clinically normal heart.

Design-Patients underwent treatment serially with verapamil (360 mg daily), sotalol (240 or $320 \mathrm{mg}$ daily), and flecainide (200 or $300 \mathrm{mg}$ daily), (the larger dose was for patients heavier than $80 \mathrm{~kg}$ ) to suppress tachycardia. Each drug was given orally for five half lives before testing.
\end{abstract}

Patients-23 patients with right VT associated with a clinically normal heart were studied.

Outcome measures-The effects of drug treatment were examined by the number of ventricular events on 24 hour Holter monitoring, and the ability of tachycardia to be induced by treadmill exercise testing (Bruce protocol) and programmed ventricular stimulation (Wellens protocol), compared with drug free baseline tests.

Setting-Patients were studied in a tertiary referral centre.

Results-All three drugs suppressed ventricular salvos $(>3,<5$ consecutive ventricular premature contractions) $(p<0.01)$ and VT $(p<0.05)$ on Holter monitoring and did not differ statistically in eifect. Exercise induced VT was also suppressed by all three drugs ( $p<0.01$ ) and of these sotaloi was the most effective although this was not statistically significant $(14 / 23$ inducible when drug free, 4/23 on flecainide, $2 / 23$ on sotalol, $5 / 23$ on verapamil). Sustained and nonsustained VT induced by programmed stimulation was also suppressed by the three drugs $(p<0.01)$ and again sotalol was the best of these though the differences did not achieve statistical significance $(17 / 23$ inducible when drug free, $4 / 17$ on flecainide, $2 / 17$ on sotalol, and $6 / 17$ on verapamil). Proarrhythmic effects of drugs were found in a few patients. There was no difference in the efficacy of the drugs in patients with histological abnormalities of the myocardium when compared with those of normal histology.

Conclusions-Ventricular tachycardia associated with a clinically normal heart can be suppressed by flecainide, sotalol, or verapamil. In individual patients sotalol was the most frequently effective drug (effective in $>89 \%$ of patients) and is a suitable choice for first line treatment.

\section{(Br Heart J 1992;68:392-7)}

Most patients presenting with ventricular tachycardia (VT) have easily detectable underlying cardiac disease, such as coronary artery disease, cardiomyopathy, and valvar or congenital heart disease. Five to 10 percent of patients, however, are reported to have no underlying heart disease as suggested by noninvasive and invasive assessment of left ventricular function and coronary anatomy. ${ }^{1-3}$ These patients do, however, exhibit variable degrees of histological abnormality on cardiac biopsy (60-90\%), including interstitial and perivascular fibrosis and vascular sclerosis, acute or subacute myocarditis, and an increase in interstitial adipose tissue..$^{4-6}$ The reports on the prognosis of such patients are contradictory. ${ }^{7-9}$ Although most authors suggest that prognosis is good, there are several who indicate that this may be a more common cause of sudden death in young patients than previously proposed. ${ }^{10-12}$ Even though treatment may not be indicated on prognostic grounds, many of these patients are severely symptomatic and require treatment to control the tachycardia. Most of the treatment data on these patients consist of short case series and anecdotal reports, ${ }^{13-15}$ and there has been no adequate study of the efficacy of antiarrhythmic drugs in controlling this form of VT. Our study examines and compares the efficacy of flecainide, sotalol, and verapamil in the treatment of VT in patients with apparently normal hearts.

\section{Patients and methods}

PATIENTS

Twenty three patients with a clinical history of sustained VT ( $>30 \mathrm{~s}$ ) and a clinically normal heart were studied. These patients came from a group of 26 consecutive patients with right ventricular tachycardia seen at our institution over two years. Two patients had had at least two of the drugs used in this study on a previous occasion and these were clinically ineffective. One patient was on amiodarone. 
These patients were excluded from our study. Patients gave informed consent to the protocol for drug testing and were enrolled in the study on the basis of the following criteria: (a) patients had no history of ischaemic heart disease or congenital cardiac abnormality. (b) All had chronic ventricular arrhythmia documented on multiple electrocardiographic leads either during a spontaneous episode or during an exercise test. Arrhythmia was defined as chronic when there was a minimum of two documented episodes at least one month apart. (c) Patients had a normal clinical examination, normal chest radiograph (cardiothoracic ratio $<50 \%$ ), and normal resting electrocardiogram (apart from $\mathrm{T}$ wave abnormalities). Patients with intraventricular conduction abnormalities, left or right ventricular hypertrophy, and prolongation of the QT interval were excluded. (d) No patient had angiographic evidence of coronary artery disease, reduced ejection fraction of the left ventricle, or abnormality of regional wall motion during left ventricular cineangiography as assessed by two independent observers.

All patients had episodes of palpitation, eight had experienced syncope and an additional 11 had had presyncope. The duration of symptoms ranged from two weeks to 20 years. The documented spontaneous arrhythmia was of right ventricular tachycardia (left bundle branch block-like morphology) in all 23 patients (QRS complex $>120 \mathrm{~ms}$ and predominently negative in lead V1, fig). The frontal plane axis of the clinical tachycardia was defined from the limb leads as leftward $\left(<-30^{\circ}\right)$, rightward $\left(>+90^{\circ}\right)$, or normal $\left(-30^{\circ}\right.$ to $\left.+90^{\circ}\right)$ from the vector perpendicular to the lead with the most isoelectric QRS complexes. The history of palpitations was suggestive of sustained episodes of VT in all patients with either documented arrhythmia, syncope, or presyncope. The episodes of clinical tachycardia were documented as sustained in 10 patients (spontaneous arrhythmia of uniform QRS morphology lasting $>30 \mathrm{~s}$ or requiring termination because of haemodynamic compromise) and non-sustained in the rest (uniform broad QRS complexes lasting

1

aVR

V1

V4

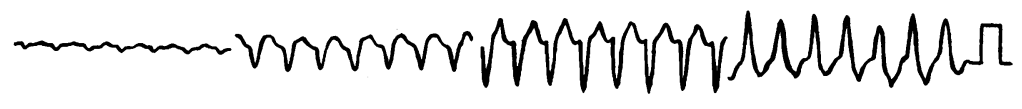

II

aVL

V2

V5

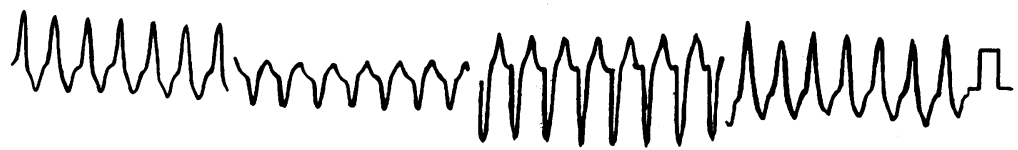

III

aVF

V3

V6

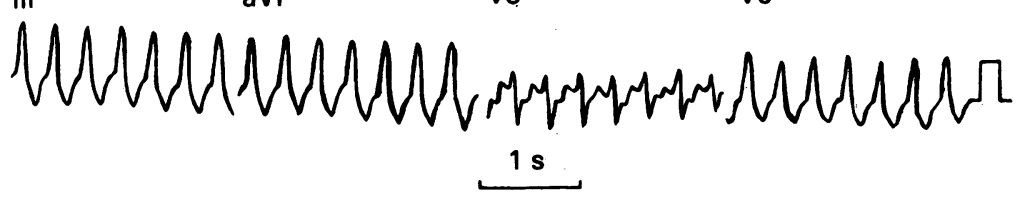

Right ventricular tachycardia with an inferior axis, typical of the form of tachycardia present in our patients.
$>3$ beats to $<30 \mathrm{~s}$ and terminating spontaneously without haemodynamic compromise). Despite the non-sustained nature of the arrhythmia in many patients, all patients were severely symptomatic and merited treatment on this basis. The mean (SD) rate of tachycardia was $212(37.8)$ beats $/ \mathrm{min}$. Patients underwent echocardiographic examination, and all had normal left ventricular function and dimensions. The findings for the right ventricular examination have been published already in a larger group of such patients. ${ }^{16}$ Signal averaged electrocardiography was performed on admission and patients also underwent full electrophysiological evaluation before entry in the study. Ventricular endomyocardial biopsies were taken and subjected to routine histopathological studies.

\section{DRUG TREATMENT}

Many of the patients had already received treatment for their tachycardia, but in all, treatment was stopped at least 72 hours before admission and evaluation of the patient in a drug free state. No patient had received amiodarone within three months of assessment. Patients received verapamil (360 mg daily in three divided doses), sotalol (240 mg or $320 \mathrm{mg}$ ), and flecainide ( 200 or $300 \mathrm{mg}$ ) daily in divided doses. The larger doses were given to patients who weighed more than $80 \mathrm{~kg}$. The drugs were given serially and maintained for a minimum of five half lives to allow stable concentrations to be reached before evaluation of efficacy. At least five half lives were allowed between drugs to wash out the previous drug. This is therefore an open, non-randomised, fixed sequence drug study. This design was accepted because of clinical and administrative limitations. A fully randomised double blind study would have required matching tablets to be given in random order. Five half lives of the drug with the longest half life would have been necessary at each stage to load and wash out the drugs. This would result in a prohibitively long admission for the patient. The use of the drugs with the shorter half lives initially allowed rapid loading and washout of the drug, reducing the time necessary for the study.

\section{EVALUATION OF TREATMENT}

Patients were evaluated with Holter monitoring, exercise testing, and programmed ventricular stimulation when drug free and on each of the three drug treatments. For drug free evaluation, the patient had been on no drugs for at least five days. On each drug, the patient was specifically asked for any subjective side effects experienced during that treatment.

\section{Holter monitoring}

Holter tapes (Tracker, Reynolds Medical Ltd, Hertford) were applied for 24 hours and analysed on a commercially available system (Pathfinder III, Mk2, Reynolds Medical). The Holter tape analysis defined the number of normal complexes, ventricular extrasystoles, couplets, salvos $(>3,<5$ consecutive ventricular premature contractions (VPCs)) and episodes of VT ( $\geqslant 5$ VPCs at $>120$ beats $/ \mathrm{min})$. 
These were then standardised to a one hour recording period before statistical analysis by non-parametric methods. The percentage suppression of ventricular extrasystoles on each treatment compared with those when drug free was also calculated. The number of patients in whom $>75 \%$ and $>90 \%$ suppression of Holter events was achieved is also given.

\section{Exercise testing}

Exercise tests and programmed ventricular stimulation (see next section) were performed two to four hours after the last dose of the drug. Treadmill exercise testing was performed with the Bruce protocol ${ }^{17}$ and the electrocardiogram was monitored during the test with apparatus from Marquette Electronics (Milwaukee, USA). Patients exercised until maximal predicted heart rate was achieved, became limited by dyspnoea and fatigue, or had sustained VT. Electrocardiographic recordings (leads II, V2, and V5) were monitored during the test and for a minimum of 10 minutes after exercise or until the cardiac rhythm returned to the pre-test sinus rate. Blood pressure and the 12 lead electrocardiogram were recorded before, and immediately after the exercise and every three min during exercise and recovery. Exercise provoked tachycardia was defined as the presence of five or more consecutive ventricular extrasystolic depolarisations occurring during or after exercise and was considered sustained if it lasted 30 seconds or longer. Changes in ST segment were monitored throughout the test, but no patient had any changes to suggest ischaemia.

\section{Programmed ventricular stimulation}

Programmed ventricular stimulation was performed after an overnight fast. After giving $10 \mathrm{ml}$ of $1 \%$ lignocaine for local anaesthesia, multipolar electrode catheters were inserted through the subclavian vein into the atrial appendange and right ventricular apex. The intracardiac electrograms and surface electrocardiograms were displayed simultaneously on a multichannel oscilloscope and recorded on a multichannel inkjet recorder (Siemens Mingograph, Solna, Sweden) at a paper speed of $25-100 \mathrm{~mm} / \mathrm{s}$. Ventricular stimulation was performed with a Medtronic 5326 programmable stimulator (Medtronic, Minneapolis, USA) with $1.8 \mathrm{~ms}$ rectangular pulses at twice diastolic threshold. Programmed ventricular stimulation was performed by the Wellens protocol. ${ }^{18}$ Briefly, one premature ventricular stimulus was introduced after every eight paced or conducted ventricular QRS complexes beginning late in diastole and then at progressively closer coupling intervals until ventricular refractoriness was encountered. The extra stimulus coupling interval was then increased by $20 \mathrm{~ms}$, out of ventricular refractoriness and a second extra stimulus was introduced. The process of diminishing coupling intervals was repeated for the second and then the third extra stimulus until a total of three extrastimuli had been introduced. Paced drive cycle lengths of 600,500 , and $400 \mathrm{~ms}$ were used. If VT was not induced by the 12 stages of Wellens protocol, isoprenaline was infused at a rate of $1-4 \mu \mathrm{g} / \mathrm{min}$ to increase the sinus rate by at least $30 \%$ or to 120 beats $/ \mathrm{min}$ (whichever was less) and the programmed ventricular stimulation was repeated. The test was considered positive if VT of the same configuration and axis as the clinically documented tachycardia was induced. If other configurations of tachycardia were induced, then the test was considered non-specific and negative. Inducible non-sustained VT was defined as ventricular extrasystoles of uniform QRS configuration, rate $>120$ beats $/ \mathrm{min}$, lasting $\geqslant 5$ beats and $<30 \mathrm{~s}$, and terminating spontaneously without haemodynamic compromise. The tachycardia was defined as sustained if it lasted $>30 \mathrm{~s}$ or required termination because of haemodynamic compromise. If the study did not induce VT in the drug free state, even after the use of isoprenaline, a further study was performed the next day. If in this study, VT could not be induced after giving isoprenaline and $120 \mu \mathrm{g}$ atropine, further programmed stimulation was not performed during the evaluation of the drugs.

\section{Statistical analysis}

Statistical analysis was performed with the SPSS package (SPSS Inc, Chicago, USA). Inducibility of the tachycardia was assessed when drug free and on each of the drug treatments for each patient. Differences between the results when drug free and during treatment with the three drugs were compared by analyses of variance, contingency tables, and non-parametric analyses as appropriate.

\section{Results}

Twenty three patients ( 12 men) mean age $42 \cdot 6$ (SD 11.8; range 21-69) years were studied. All patients had right VT of which 11 had a rightward axis, four had a leftward axis, and eight had a normal axis. The mean (SD) doses of the drugs given were 233 (64.3) mg flecainide, $270(48.2) \mathrm{mg}$ sotalol, and $360(0.0) \mathrm{mg}$ verapamil. Table 1 shows the results of the Holter monitoring. There was a large individual variability in the number of ventricular extrasystoles in the 24 hours, but the effects of the different drug treatments were fairly consistent. All three drugs resulted in partial suppression of ventricular extrasystoles, couplets, salvos, and episodes of VT on Holter monitoring. The numbers of patients with $90 \%$ and $100 \%$ suppression of VT on Holter monitoring were similar on the three drugs (table 1). The drugs did not differ statistically in efficacy. The comparative ineffectiveness of flecainide in reducing Holter monitored events contrasts with previous reports of its efficacy in other types of VT. An increase in the hourly episodes of VT $>150 \%$ over drug free values was seen in one patient on flecainide, one patient on sotalol, and two patients on verapamil.

Table 2 gives the results of exercise testing The time to the end of the test, whether this was by exhaustion, achievement of the maximum heart rate, or start of tachycardia, was not affected by any of the treatments. The time to 
Table 1 Results of Holter monitoring and the number of patients with suppression of Holter monitored events when drug free and on three treatments

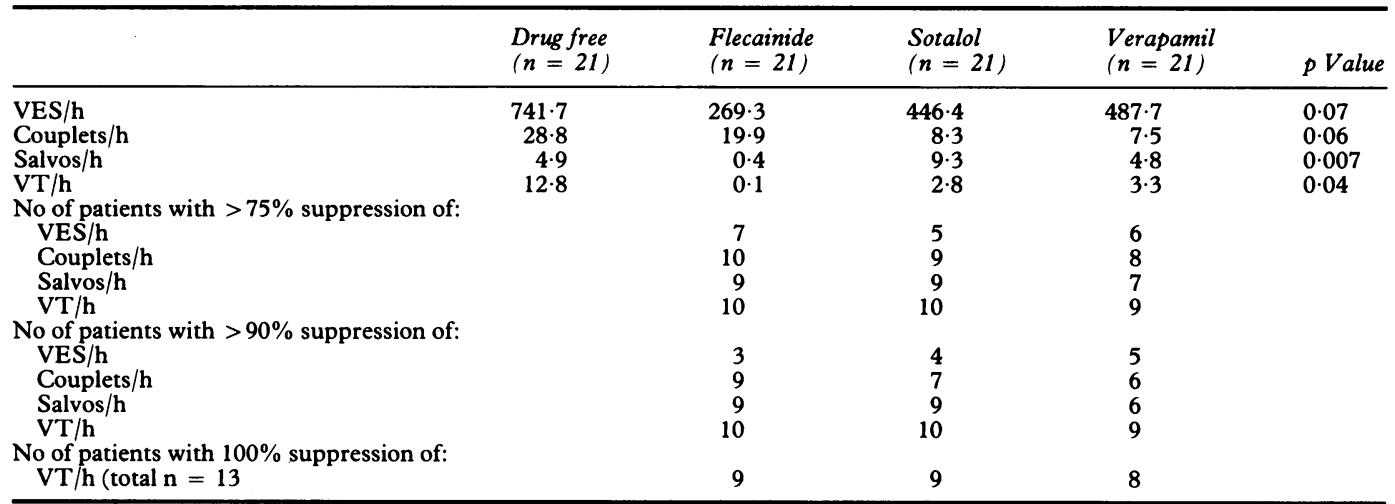

Data are on 21 patients because of tape failures on two. VES, ventricular extrasystoles.

the end of the test on sotalol did not differ significantly from the other treatments although it was a little lower. Fourteen patients developed exercise induced VT when drug free and in four the arrhythmia was sustained. All three drugs resulted in some suppression of exercise induced VT and there were only two episodes of sustained tachycardia, one on sotalol and one on verapamil. Two patients on flecainide, one patient on sotalol, and three patients on verapamil had VT at exercise on the drug when there had been no arrhythmia when drug free (table 3). This may be due to the inherent variability of induction of arrhythmia with exercise or a genuine proarrhythmic effect. Sotalol was the best of the three drugs but this difference was not significant $(p=0 \cdot 4)$. The stage at which tachycardia was induced was not significantly influenced by the treatments ( $\mathrm{p}=0.4$ for all treatments), though more patients had tachycardia during the test rather than in recovery on flecainide and verapamil when compared with sotalol.

Tachycardia was induced by programmed ventricular stimulation in 17 patients when drug free, in all cases only tachycardia that was of similar configuration and axis to the clinical tachycardia was considered relevant. In one patient the clinical tachycardia was only stimulated after isoprenaline infusion $(2 \mu \mathrm{g} /$ min) when drug free and the same dose of isoprenaline was used on all the subsequent trials with antiarrhythmic treatment. In seven patients, the induced tachycardia was sustained. Inducible tachycardia was rendered non-inducible by all three drug treatments in over $50 \%$ of the patients (table 4 ). Although flecainide when compared with sotalol made fewer patients non-inducible (13/17 $v$ 15/17)

Table 2 Results of exercise testing when drug free and with three drug treatments

\begin{tabular}{lcccc}
\hline & $\begin{array}{c}\text { Drug free } \\
(n=23)\end{array}$ & $\begin{array}{l}\text { Flecainide } \\
(n=23)\end{array}$ & $\begin{array}{l}\text { Sotalol } \\
(n=23)\end{array}$ & $\begin{array}{l}\text { Verapamil } \\
(n=23)\end{array}$ \\
\hline Exercise tolerance & 599 & 627 & 617 & 643 \\
(SD) & $(199)$ & $(169)$ & $(201)$ & $(153)$ \\
Non-inducible $_{\text {Inducible }}^{\star}$ & 10 & 20 & 22 & 19 \\
Sustained tachycardia $^{\star}$ & 14 & 4 & 2 & 5 \\
p Value† & 4 & 0 & 1 & 1 \\
\hline
\end{tabular}

$\star$ Number of patients.

†For differences between the drug treatments and drug free for induction of ventricular tachycardia (VT). and non-sustained $(2 / 7 v 6 / 7)$ these differences were not significant $(\mathrm{p}=0.4$ and $\mathrm{p}=0.9)$. More patients were inducible (6/17) and sustained $(4 / 7)$ on verapamil when compared with sotalol ( $p=0.07$ and $p=0.8$ respectively). If the induction and suppression of only sustained VT was considered, the results were similar and all three drugs suppressed inducible VT, but did not achieve statistical significance because of the small numbers. The stage of the Wellens protocol at which tachycardia was induced was also increased by all three treatments $(p<0.01)$ but no difference could be shown between the treatments ( $p=0.4$; data not presented). Two patients on flecainide, one patient on sotalol, and three patients on verapamil developed sustained VT on the drug, whereas VT in these patients had been non-sustained when drug free. In one patient the induced tachycardia was faster on sotalol than the drug free arrhythmia and required cardioversion, whereas two of the tachycardias induced on verapamil were haemodynamically unstable, requiring cardioversion. Side effects from the drugs included five patients who experienced dizziness on flecainide and six said they were tired on sotalol. Two patients had some tiredness on verapamil. Individual patients responding to one drug, in general responded to the other drugs and there was no difference in the efficacy of the drugs in patients with abnormal histology compared with those with normal histology in any of the three tests.

\section{Discussion}

The results suggest that right VT associated with a normal heart responds well to the drugs

Table 3 Number of patients with or without ventricular tachycardia (VT) during exercise when drug free and in three treatment groups

\begin{tabular}{lccc}
\hline & Flecainide & Sotalol & Verapamil \\
\hline No VT baseline & 7 & 8 & 6 \\
No VT drug & 12 & 13 & 12 \\
VT baseline & 2 & 1 & 2 \\
No VT drug & 2 & 1 & 3 \\
VT baseline & VT drug & & \\
No VT baseline & &
\end{tabular}


Table 4 Results of programmed stimulation when patients were drug free and in each of three treatments groups

\begin{tabular}{lcccc}
\hline & $\begin{array}{c}\text { Drug free } \\
(n=23)\end{array}$ & $\begin{array}{c}\text { Flecainide } \\
(n=17)\end{array}$ & $\begin{array}{c}\text { Sotalol } \\
(n=17)\end{array}$ & $\begin{array}{c}\text { Verapamil } \\
(n=17)\end{array}$ \\
\hline $\begin{array}{l}\text { Non-inducible } \\
\text { Inducible }\end{array}$ & 6 & 13 & 15 & 11 \\
$\begin{array}{l}\text { Sustained tachycardia } \\
\text { Mean rate of tachycardia } \dagger\end{array}$ & 17 & 4 & 2 & 6 \\
$\begin{array}{l}\text { (beats/min) } \\
\text { Value } \neq\end{array}$ & 7 & 2 & 1 & 4 \\
\hline
\end{tabular}

^Number of patients; †rate during induced sustained VT; $\ddagger p$ value for differences between drug treatment and drug free for induction of tachycardia.

tested in this study. Although differences between the drugs did not achieve significance, sotalol was generally the most effective.

Earlier studies of the treatment effects of drugs in VT associated with a normal heart are few. Buxton et al examined the effect of antiarrhythmic drugs upon spontaneous arrhythmia in right ventricular tachycardia and reported that class 1 antiarrhythmic agents (procainamide, quinidine) suppressed all VT in 9/16 patients and were ineffective in seven. ${ }^{3}$ Propranolol suppressed spontaneous VT in 8/ 16 patients whereas verapamil was effective in 1/4. Amiodarone was used in 2/4 patients and two were not suppressed by any form of drug treatment. Ventricular tachycardia inducible by programmed stimulation was rendered noninducible in 8/9 patients by class 1 agents, in $3 / 6$ patients by propranolol, and in $0 / 4$ patients by verapamil. Rahilly et al reported a series of patients with repetitive monomorphic VT associated with a normal heart, which included patients with VT of right and left ventricular origin in whom $7 / 7$ patients responded to enacainide. ${ }^{19}$ Five other patients received either quinidine or propranolol singly, combined, or aprinidine. Six patients with right ventricular outflow tachycardia reported by Pietras et al were evaluated by serial treadmill testing. ${ }^{13}$ In these procainimide, quinidine, and disopyramide were relatively ineffective whereas propranolol prevented treadmill provocation of VT in all patients. Similar results were obtained by Brodsky et al where in six patients, treatment with $\beta$ blocker prevented induction of VT by exercise and programmed stimulation and reduced ventricular extrasystoles and VT on Holter monitoring. ${ }^{14}$ Lemery et al in their series of VT of right and left ventricular origin reported that most patients $(48 \%)$ were treated with either sotalol or a class IC agent, whereas amiodarone was used in $19 \%$, and a calcium channel blocker was used in two patients. ${ }^{9}$ In a specific study of right ventricular tachycardia, sotalol was reported as effective as were class IC agents, whereas verapamil was effective in only one patient. ${ }^{15}$ These studies contrast with the present report where a single configuration of VT has been studied and preselected drugs used at fixed doses.

Our study is not a randomised controlled trial, and therefore, it is difficult to ascertain placebo effects, elements of bias, and carry over effects. Although it is straightforward to organise and conduct a study of two drugs in a double blind placebo controlled manner, a trial involving three drugs and a placebo presents con- siderable logistic difficulties. The study does not include drug concentrations and although sotalol and flecainide have somewhat long half lives, that of verapamil is variable. Furthermore, verapamil is subject to substantial first pass metabolism. To minimise variation due to these factors, exercise tests and programmed stimulation were performed two to four hours after the last drug dose. The tests used for evaluation of drug efficacy in this study are not subject to bias, and adequate periods were allowed for the drug concentrations to equilibrate and wash out. This study, despite its deficiencies, is the only systematic study of drug efficacy in the suppression of VT associated with a normal heart. A study of this nature does, therefore, provide valuable information allowing appropriate choice of drug for patients with this form of tachycardia.

All patients had left bundle branch blocklike morphology VT, which can usually be mapped to the right ventricle ${ }^{3}$ and is often from the region of the outflow tract when associated with a rightward or normal axis. Electrophysiological mapping of the tachycardia of patients in this study was performed in eight cases and this showed the origin of the tachycardia to be in the right ventricle or the septal aspect of the right ventricular outflow tract in seven subjects and in the free wall of the outflow tract in one. Our experience with these forms of tachycardia is similar to that of other groups in that they are often inducible by exercise and less frequently by programmed ventricular stimulation. ${ }^{7-9}$

Whether this form of tachycardia requires treatment, and the prognosis of VT associated with a normal heart remain controversial issues. Many studies suggest that when VT occurs in association with a normal heart prognosis is good and the patients do not suffer sudden death. ${ }^{278}$ This contrasts with VT associated with ischaemic heart disease and cardiomyopathy where there is a high mortality associated with symptomatic VT. There are, however, several studies reporting that prognosis of VT in young patients with "normal" hearts may be worse than previously proposed. ${ }^{10-12}$ Many of the patients in these groups had frank arrhythmogenic right ventricular dysplasia, and most had right VT. Though none of our patients had obvious evidence of arrhythmogenic right ventricular dysplasia, many patients showed evidence of endocardial and interstitial fibrosis, and some had fatty infiltration of the myocardium, which may represent variant forms of this condition. Future studies need to concentrate on the classification of VT associated with a normal heart and the natural history of the tachycardia in these subgroups. In our own series of 40 patients with VT associated with a normal heart followed up for a period of three years, there have been two deaths, one related to surgery for incessent VT, and the other a sudden death, presumably related to arrhythmia. This mortality is higher than that expected in such a young group of subjects suggesting that the condition may not be as benign as reported in previous studies. 
Although many patients may be more or less symptom free some have severe symptoms and suffer disabling attacks of palpitation. All the patients in this study had evidence of sustained episodes of VT with symptoms of sustained palpitation, syncope, or presyncope related to arrhythmia. Thus although some patients with only non-sustained episodes of palpitation may not require drug treatment, many will have disabling symptoms necessitating treatment. Our study and the clinical experience of other groups suggest that these tachycardias are suppressed by the $\beta$ blocking and class III activity of sotalol, the class IC effect of flecainide and the class IV action of verapamil. In this study the effect of a pure $\beta$ receptor blocking agent was not examined, but there are three previous reports of its efficacy. ${ }^{131420}$ Overall, sotalol was the most effective drug in suppression of the tachycardia in these patients, although flecainide was similar. Verapamil was not as effective as the other two agents.

Although there is variability of the response to the test used to assess efficacy of treatment, our study appears to show that proarrhythmic effects may occur in this patient group; some patients developed exercise induced VT whereas these had been free of arrhythmia when drug free; some with non-sustained VT when drug free became sustained and haemodynamically unstable on the drug during programmed ventricular stimulation. Patients will therefore require careful assessment before the introduction of long-term drug treatment. Patients who responded to one drug were, in general, responsive to the other agents used in the study. There were, however, some patients in whom one agent was effective whereas there was no response to the others. Sotalol was well tolerated as treatment in the group as a whole with few side effects. It therefore seems reasonable to treat such patients with sotalol as first line treatment and use other agents only when this fails. The responsiveness of some of the patients in this group to verapamil is interesting as it suggests that some of these tachycardias may be due to triggered activity rather than to a re-entry mechanism. ${ }^{21}$ There are no publications concerned with how efficacy of drugs should be assessed in this group. Our data suggest that patients who have therapeutic suppression of exercise induced VT, and VT induced by programmed stimulation, also have good control of Holter monitored events and symptoms, suggesting that all three methods of monitoring efficacy may play a part in the mangement of such patients.

We conclude that VT associated with a normal heart responds well to all three forms of drug treatment used in this study. The most efficacious agent for suppression of exercise induced and VT induced by programmed ventricular stimulation appears to be sotalol. Flecainide and verapamil can be used in cases where sotalol has failed.

1 Froment R, Gallavardin L, Cahen P. Paroxysmal ventricular tachycardia: a clinical classification. Br Heart J 1953;15: tachycard

2 Chapman JH, Schrank JP, Crampton RS. Idiopathic ventricular tachycardia. An intracardiac electrical, hemotricular tachycardia. An intracardiac electrical, hemoJ Med 1975;59:470-80.

3 Buxton AE, Waxman HL, Marchlinski FE, Simson MB, Cassidy D, Josephson ME. Right ventricular tachycardia: Clinical and electrophysiological characteristics. Circulation 1983;68:917-27.

4 Strain JE, Grose RM, Factor SM, Fisher JD. Results of endomyocardial biopsy in patients with spontaneous ventricular tachycardia but without apparent heart disease. Circulation 1983;68:1171-81.

5 Sugrue DD, Holmes DR, Gersh BJ, Edwards WD, McLaran CJ, Wood DL, et al. Cardiac histologic findings
in patients with life threatening ventricular arrhythmias of unknown origin. J Am Coll Cardiol 1984;4:952-7.

6 Metha D, McKenna WJ, Ward DE, Davies MJ, Camm AJ. Significance of signal-averaged electrocardiography in relation to endomyocardial biopsy and ventricular relation to endomyocardial biopsy and ventricular
stimulation studies in patients with ventricular tachystimulation studies in patients with ventricular tachy-
cardia without clinically apparent heart disease. J Am Coll cardia without clinically

7 Holt PM, Wainwright RJ, Curry PVL. Right ventricular outflow tract tachycardias in patients without apparent structural heart disease. Int J Cardiol 1986;10:99-110.

8 Deal BJ, Miller SM, Scagliotti D, Prechel D, Gallastegui JL, Hariman RJ. Ventricular tachycardia in a young population without overt heart disease. Circulation 1986; 73:1111-8.

9 Lemery R, Brugada P, Bella PD, Dugernier T, van den Dool A, Wellens HJJ. Nonischaemic ventricular tachycardia. Clinical course and long-term follow-up in patients without clinically overt heart disease. Circulation 1989;79.

Rowland TW, Schweiger MJ. Repetitive paroxysmal ventricular tachycardia with sudden death in a child. $A m \mathrm{~J}$ Cardiol 1984;53:1729.

11 Benson DW, Benditt DG, Anderson RW, Dunnigan A, Pritzker MR, Kudik TJ, Zavorel JH. Cardiac arrest in young ostensibly healthy patients. Clinical hemodynamic and electrophysiologic findings. Am J Cardiol 1983;52: 65-9.

12 Theine G, Nava A, Corrodo D, Rossi L, Pennelli N. Right ventricular cardiomyopathy and sudden death in young people. N Engl J Med 1988;3:129-33.

13 Pietras RJ, Lam W, Bauernfeind R, Sheikh A, Palileo E, Strasberg B, et al. Chronic recurrent right ventricular tachycardia in patients without ischemic heart disease: Clinical, hemodynamic, and angiographic findings. $\mathrm{Am}$ Heart $J$ 1983;105:357-66.

14 Brodsky MA, Sato DA, Allen BJ, Chesnie BM, Henry WL. Solitary beta-blocker therapy for life-threatening ventricular tachyarrhythmias. Chest 1986;89:790-4.

15 Proclemer A, Ciani R, Feruglio GA. Right ventricular tachycardia with left bundle branch block and inferior axis morphology: clinical and arrhythmological characteristics morphology: clinical and arrhythmologica

16 Mehta D, Odawara H, Ward DE, McKenna WJ, Davies MJ, Camm AJ. Echocardiographic and histologic evaluation of the right ventricle in ventricular tachycardias of left bundle branch block morphology without overt cardiac abnormality. Am J Cardiol 1989;63:939-44

17 Bruce RA, Hornsten TR. Exercise stress testing in evaluation of patients with ischemic heart disease. Prog Cardiovasc Dis 1969;11:371-90.

18 Wellens HJJ, Brugada P, Stevenson WG. Programmed electrical stimulation of the heart in patients with lifethreatening ventricular arrhythmias. What is the significance of induced arrhythmias and what is the correct stimulation protocol? Circulation 1985;72:1-7.

19 Rahilly GM, Prystowsky EN, Zipes DP, Naccarelli GV, Jackman WM, Heger JJ. Clinical and electrophysiologic findings in patients with repetitive monomorphic ventricular tachycardia and otherwise no
diogram. Am J Cardiol 1982;50:459-68.

20 Palileo EV, Ashley WW, Swiryn S, Buernfeind RA, Strasberg B, Petropoulos T, Rosen KM. Exercise provocable berg B, Petropoulos T, Rosen KM. Exercise provocable right ventricular
$1982 ; 104: 185-93$.

21 Lerman BB, Belardinelli L, West A, Berne RM, DiMarco JP. Adenosine-sensitive ventricular tachycardia: evidence suggesting cyclic AMP-mediated triggered activity. Circulation 1986;74:270-80. 